\title{
Labyrinthe
}

20 | 2005 (1)

La Cognition

\section{Musique et sciences cognitives}

\section{Michel Gribenski}

\section{(2) OpenEdition}

Journals

Édition électronique

URL : http://journals.openedition.org/labyrinthe/763

DOI : $10.4000 /$ labyrinthe.763

ISSN : 1950-6031

Éditeur

Hermann

Édition imprimée

Date de publication : 20 avril 2005

Pagination : 99-116

Référence électronique

Michel Gribenski, « Musique et sciences cognitives », Labyrinthe [En ligne], 20 | 2005 (1), mis en ligne le 08 juillet 2008, consulté le 19 avril 2019. URL : http://journals.openedition.org/labyrinthe/763 ; DOI :

$10.4000 /$ labyrinthe.763

Propriété intellectuelle 


\title{
MUSIQUE ET SCIENCES COGNITIVES
}

\author{
Michel Gribenski \\ gribenski@hotmail.com
}

« [...] me donnerais-je la peine de parler, si une lucarne s'ouvrait sur mon front, devant ma poitrine, en sorte que la personne à qui je veux énumérer une à une mes pensées, offrir un à un mes sentiments, pût toujours savoir longtemps à l'avance où j'allais en venir? »

GOETHE, Les Affinités électives, $1^{\text {re }}$ partie, chapitre 4 (traduit par Pierre du Colombier)

\section{Prêter l'oreille}

Comment entend-on? La question paraît paradoxale : il semble tout simplement que les oreilles perçoivent des sons, et que ce processus de perception concerne l'acoustique - neuro-acoustique et psychoacoustique. Certes. Mais encore faut-il distinguer perception des phénomènes acoustiques et organisation de cette information acoustique en représentations sonores cohérentes et hiérarchisées: c'est ce que différencie l'anglais avec audition et hearing - en français, le couple entendre/écouter ou audition/écoute est impropre à rendre cette distinction, car il ne s'agit pas d'une différence d'attention, mais d'une différence entre processus physiologique, nerveux d'une part, et cognitif d'autre part. Or, c'est bien à la façon dont nous interprétons les données de notre perception que s'intéressent les sciences cognitives. Après s'être demandé comment on entend la musique (limitée ici à la musique occidentale), on se demandera pourquoi l'on a du mal à entendre la musique contemporaine savante, tout particulièrement la musique sérielle des années 1950. Un demi-siècle après la création du Marteau sans maître (1955) de Pierre Boulez (dont on fête cette année 
le quatre-vingtième anniversaire), il m'a en effet semblé qu'il fallait prêter l'oreille à cette question: qu'est-ce qui, dans la musique sérielle - et peut-être plus généralement atonale ${ }^{1}$ - passerait les limites cognitives de notre entendre?

Pourquoi se limiter ici à la seule musique occidentale savante, à l'exclusion des musiques occidentales populaires et des musiques non occidentales? Outre qu'il fallait bien restreindre un champ d'investigation déjà immense, il m'a semblé que ces musiques n'avaient pas cherché, dans l'ensemble, à excéder le système musical général dans lequel elles s'inscrivaient - tonal ${ }^{2}$ pour les musiques populaires occidentales, diversement modales ${ }^{3}$ selon les musiques non occidentales concernées ${ }^{4}$. C'est peut-être l'un de leurs mérites - c'est aussi l'une de leurs limites, que de ne pas chercher, précisément, à explorer les limites, fussent-elles indépassables, sous peine de provoquer de graves malentendus. Mais qui a dit que les malentendus n'étaient pas féconds? Il est peut-être nécessaire de brouiller momentanément l'écoute pour mieux entendre.

\footnotetext{
1. J'expliquerai plus bas, au seuil de la seconde partie de cet article, ce qu'on peut entendre par « musique sérielle » et par « musique atonale »; qu'il suffise pour l'instant d'indiquer que la musique sérielle est un type organisé de musique atonale.

2. «Tonal» - on y reviendra - renvoie à un système mélodique, harmonique et métrique hiérarchisé, fondé sur les modes majeur et mineur et sur une métrique régulière organisant le rythme. L'idée d'une référence exclusive de la musique populaire occidentale récente au système tonal doit être nuancée: des recherches expérimentales ont été menées dans le domaine du rock (Can, King Crimson) ou du free-jazz (Ornette Coleman, dans une moindre mesure John Coltrane) - même si le rattachement du free-jazz à la musique populaire est également problématique... Je remercie Dimitri Champain pour ses précisions sur ce point, et plus généralement pour ses suggestions, critiques et idées pleines de bon sens.

3. La musique modale, largement dominante dans le temps et dans l'espace, est celle qui repose sur des modes, c'est-à-dire sur des gammes ou échelles mélodiques organisées (autres que les deux modes majeur et mineur, qui définissent le système de la tonalité); ces gammes étaient liées originellement à des formules mélodiques typiques. En occident, les modes ont été développés dans l'Antiquité et au moyen âge, puis repris (même s'ils n'avaient jamais totalement disparu) au Xx ${ }^{\mathrm{e}}$ siècle, soit comme enrichissement de la tonalité (chez Debussy par exemple), soit comme système d'organisation mélodico-harmonique (chez Messiaen notamment). Hors Occident, l'ensemble des musiques existantes repose sur des modes très diversifiés: «Un mode suppose une couleur, un caractère musical particulier, une expressivité qui soit spécifique. Les musiques traditionnelles du monde entier utilisent fréquemment des systèmes musicaux fondés sur des conceptions modales extrêmement variées; les modes employés le sont alors selon des règles propres à chacun de ces musiques. » (Claude Abromont, Eugène de Montalembert, Guide de la théorie de la musique, Paris, Fayard, p. 543).

4. Je reviendrai brièvement, dans le cours de cet article, sur la question des musiques non occidentales, pour l'examen de l'argument que j'appelle «naturaliste».
} 


\section{L'oreille et l'esprit musiciens Construction, élaboration}

L'écoute de la musique est le fruit d'une construction, d'une élaboration $^{5}$ : ainsi, les sciences cognitives cherchent à mettre au jour les processus mentaux selon lesquels l'esprit organise le donné sensationnel en une représentation cohérente. La psychologie cognitive le fait à travers un ensemble d'expériences menées comparativement sur différents types de sujets. À cet égard, le titre de l'ouvrage fondateur de John A. Sloboda en 1985 : The Musical Mind [L'Esprit musicien $]^{6}$ fait figure d'emblème: ce ne sont pas seulement les oreilles qui perçoivent, mais l'esprit qui élabore et construit des représentations mentales, lesquelles, loin de se réduire aux aspects physiologiques et psycho-acoustiques de l'audition, sont organisées en un système culturel, symbolique et esthétique. "La plupart des auditeurs possèdent et utilisent des processus perceptifs et cognitifs hautement sophistiqués pour comprendre, apprécier et participer aux activités musicales »: ainsi Stephen McAdams et Emmanuel Bigand définissent-ils cet «esprit musicien ${ }^{7} »$. Certes, les sciences cognitives s'inscrivent dans l'héritage de la psychologie de la musique $^{8}$ et de la psycho-acoustique, mais la radicalité du questionnement qu'elles apportent renouvelle en profondeur l'approche de l'expérience musicale.

Les spécialistes de la cognition musicale (en particulier Stephen McAdams et Emmanuel Bigand, qui s'appuient notamment sur les travaux générativistes de Fred Lerdahl et Ray Jakendorff 9 ) ont ainsi construit un modèle d'écoute en cinq étapes successives ${ }^{10}: 1$ ) transfor-

\footnotetext{
5. Apparemment synonymes, ces deux termes ont en réalité des connotations fort différentes, parallèles à la distinction que l'on fait, au sein des sciences cognitives, entre «cognitivisme» et « connexionnisme». Je n'entrerai pas ici dans ce débat complexe, qui engage les présupposés théoriques fondamentaux de la discipline, ainsi que sa dimension historique.

6. L'Esprit musicien. La psychologie cognitive de la musique, 1988 [1985].

7. Emmanuel Bigand, Stephen McAdams, éd., Penser les sons. Psychologie cognitive de la musique, 1994, p. 5.

8. Voir en particulier Robert Francès, La Perception de la musique (Paris, Vrin, 1984 [1958]), Paul Fraisse, Psychologie du temps (Paris, Puf, 1967) et La Psychologie du rythme (Paris, Puf, 1974), ainsi que Michel Imberty, L'Acquisition des structures tonales chez l'enfant (Paris, Klincksieck, 1969). 9. Fred Lerdahl et Ray Jackendorff, A Generative Theory of Tonal Music, Cambridge, Mass., MIT Press, 1983.

10. Je reprends ici le modèle proposé par Emmanuel Bigand, dans Emmanuel Bigand, Stephen McAdams, éd., op. cit., ch. 8: «Contributions de la musique aux recherches sur la cognition auditive humaine », p. 267 et suivantes.
} 
mation, au niveau du système auditif, des vibrations aériennes en impulsions nerveuses ; 2) traitement de l'information acoustique organisée en images auditives cohérentes; 3) segmentation de la surface musicale en laps de temps ${ }^{11}$; 4) établissement d'une hiérarchie d'événements; 5) perception de schémas de tensions et de détentes musicales. L'un des prolongements possibles de la modélisation des processus d'écoute concerne la valeur symbolique attribuée aux différentes musiques: selon quels processus cognitifs et quels codes culturels, des affects notamment sont-ils associés à telle ou telle musique?

\section{Inné/acquis}

L'un des enjeux essentiels des recherches cognitivistes est la discrimination entre aptitudes innées et compétences acquises, d'où l'importance accordée au processus d'apprentissage chez l'enfant. Le fait que la perception musicale relève d'une construction ne signifie pas qu'il n'y ait pas de dispositions innées ou, corrélativement, d'universaux musicaux. Mais ces éventuelles dispositions innées doivent être activées par des processus d'apprentissage qui, eux, sont culturels: il y a une dialectique de l'inné et de l'acquis. Ainsi, l'oreille absolue, faculté de percevoir la hauteur absolue des notes qui n'est développée que par certains sujets, semble être un phénomène de mémoire à long terme.

\section{Mémoire}

La mémoire joue, en effet, un rôle essentiel dans le traitement de l'information acoustique, avec différents types de mémoires à court, moyen et long termes. Si la mémoire à court terme intervient au moment même de l'écoute, permettant au sujet de construire une image sonore cohérente, la mémoire à moyen terme permet d'identifier, à l'échelle d'un morceau, des motifs et des caractères récurrents ainsi que leurs transformations éventuelles (dans le cas de variations, par exemple); enfin, c'est la mémoire à long terme qui structure et organise l'esprit musicien - que le sujet soit, lui, musicien ou non: de nombreuses expériences ont prouvé, par exemple, la connaissance implicite des différentes tonalités chez tous les sujets occidentaux non

11. Cette segmentation, précise Bigand, se fait conformément aux principes de groupement établis par la théorie de la forme ou Gestalttheorie (Emmanuel Bigand, Stephen McAdams, éd., op. cit. p. 271). 
musiciens, et montré plus généralement qu' «il existe dans toute culture musicale des configurations rythmiques, mélodiques, ou rythmicomélodiques, qui apparaissent assez fréquemment pour que les auditeurs puissent les avoir stockées en mémoire à long terme sous forme d'un lexique de schémas et de formes prototypiques ${ }^{12} »$.

Les sciences cognitives, approfondissant les acquis de la psychoacoustique et de la psychologie expérimentale, ont ainsi contribué à mettre au jour les processus mentaux impliqués par la perception, l'exécution et la composition musicales ${ }^{13}$. Elles montrent que l'expérience musicale est, en partie, une construction ou une élaboration, faisant intervenir de façon centrale la mémoire, que ce soit dans la reconnaissance des différents paramètres musicaux, dans leur hiérarchisation ou dans leur organisation dans le temps. Il reste à poursuivre dans cette voie, afin, peut-être, de parvenir à une modélisation, plus complète encore, de l'oreille et de l'esprit musiciens.

Or, on s'aperçoit que certaines musiques contemporaines restent, même un demi-siècle après leur création, très difficiles d'écoute: les sciences cognitives peuvent-elles nous dire quelque chose de ce malentendu?

\section{Composition musicale et cognition ${ }^{14}$}

«Cessez de brouiller l'écoute!» Invectives drolatiques d'un mélomane aux compositoires les plus notoires (anonyme, début du XXI ${ }^{\mathrm{c}}$ siècle)

\section{Malentendu}

Un malentendu n'est jamais anodin: il est le signe d'un problème qui doit être interrogé, analysé, posé, c'est-à-dire en premier lieu

12. Emmanuel Bigand, dans Emmanuel Bigand, Stephen McAdams, éd., op. cit., p. 261-262.

13. Faute de place et en raison de ma problématique centrée sur la question de l'audition, j'ai laissé de côté l'analyse cognitive de l'exécution et, en partie, celle de la composition (et de l'improvisation) musicales: on se reportera, pour ces questions, à John A. Sloboda, L'Esprit musicien. La psychologie cognitive de la musique, op. cit., chapitres 3 et 4.

14. Sur cette question, voir essentiellement Composition et perception, dans Contrechamps, $\mathrm{n}^{\circ} 10$, L'Âge d'homme, 1989, et John A. Sloboda, L'Esprit musicien. La psychologie cognitive de la 
entendu - sous peine de provoquer une mésentente, voire une brouille. Bien entendu, ce problème revêt en musique une gravité (ou une acuité) particulière. Or, c'est bien sous le signe du malentendu - entre compositeurs et public - qu'est placée, depuis le début des années 1950, la musique occidentale savante. Combien de mélomanes avertis déclarent, en effet, " aimer la musique de Bach à Stravinski, à Ravel ou à Richard Strauss »... mais ne rien entendre (ne rien comprendre, ne rien aimer) à/de la musique de Schönberg, de Webern, de Boulez... Manque d'acculturation du public (nourri à la musique tonale) à un système nouveau, celui de l'atonalité (dodécaphonique, sérielle ou autre) ? C'est ce qu'avaient répondu par avance les avant-gardistes, signalant qu'il ne s'agissait pas du premier malentendu de l'histoire de la musique. Le problème est que le malentendu a la dent dure, et dure depuis un demisiècle... Peut-il être interprété, à la lumière des sciences cognitives, comme le symptôme d'un mal plus grave: comme un mal entendre, au sens d'un mal comprendre, qui engendrerait un mal aimer?

Pour réfléchir sur cette question, je m'appuierai essentiellement sur un article de Fred Lerdahl intitulé "Contraintes cognitives sur les systèmes compositionnels ${ }^{15}$ ». Coauteur, avec Ray Jackendorff, d'une Théorie générative de la musique tonale ${ }^{16}$, Lerdahl se pose, dans cet article, la question de savoir «pourquoi [même] les auditeurs compétents n'entendent pas les séries quand ils écoutent de la musique sérielle ${ }^{17} \gg$.

Mais auparavant, plusieurs mises au point préalables sont nécessaires pour éviter d'ajouter de la confusion au malentendu.

\footnotetext{
musique, op. cit., p. 145-206. Citons pour mention le livre récent de Jocelyne Kiss : Composition musicale et sciences cognitives. Tendances et perspectives, Paris, L'Harmattan, « Univers musical », 2004, qui ne problématise pas la question de façon très satisfaisante.

15. Article paru dans Composition et perception, op. cit., p. 25-57. On aura grand profit à prendre également connaissance, dans le même numéro de la revue Contrechamps, de la controverse ayant opposé le musicologue et sémioticien Jean-Jacques Nattiez et le sociologue Pierre-Michel Menger sur le divorce entre compositeurs et public dans la musique contemporaine: Jean-Jacques Nattiez, « Le paradoxe du sociologue. Esthétique et perception musicales dans les travaux de Pierre-Michel Menger » (p. 140-169) et Pierre-Michel Menger, «Jugement scientifique et soupçon critique. Réponse à Jean-Jacques Nattiez » (p. 170-176). Voir également Robert Francès : « La perception de la musique sérielle», dans La Perception de la musique, op. cit., p. 133-144.

16. Op. cit. supra (note 9).

17. Fred Lerdahl, art. cité, p. 48
} 


\section{Musiques tonale, atonale, sérielle}

Tout d'abord, pour la clarté du débat, il faut définir, rapidement, ce que l'on entend ici par musique sérielle, par rapport notamment à musique atonale. En schématisant à l'extrême, l'histoire de la musique occidentale peut être lue comme la construction, au moyen âge, d'un système modal fondé sur de nombreuses gammes mélodiques et modes rythmiques; puis, à partir du XVII ${ }^{\mathrm{e}}$ siècle, par celle d'un système tonal, mélodico-harmonique, par sélection de deux modes appelés majeur et mineur, associés à une métrique régularisant le rythme; enfin, par la déconstruction, au tournant des XIX ${ }^{\mathrm{e}}$ et $\mathrm{XX}^{\mathrm{e}}$ siècles, du système tonal au profit d'un système atonal, puis dodécaphonique et sériel. (Il importe de ne pas faire de ces moments - modal, tonal, atonal - autre chose que des repères dans l'histoire de la musique : à l'ère de la musique atonale, ont continué d'exister maintes musiques tonales - la musique atonale étant même demeurée un minuscule îlot dans l'océan tonal que constituent les musiques populaires et même parfois savantes - que l'on songe à Britten, à Chostakovitch, à Prokofiev, à Dutilleux, etc. C'est précisément cette linéarité historique hégélienne que critiquent ceux qui remettent en cause la nécessité atonale.) Schönberg tira de l'évolution du langage musical à la fin du XIX ${ }^{\mathrm{e}}$ siècle, avec l'extension du chromatisme, notamment chez Wagner, la conclusion qu'il était nécessaire de sortir du langage tonal: après une période dite $\mathrm{d}$ ' «atonalité libre », il décida de créer un nouveau système musical fondé, non plus sur les gammes majeure et mineure, mais sur les douze degrés chromatiques, ces derniers étant organisés, non de façon hiérarchique, mais selon des séries appelées, en raison du nombre de demi-tons les composant, «dodécaphoniques». L'extension de la série des hauteurs mélodiques aux intervalles et aux autres paramètres du son (durée, intensité, timbre), inaugurée par Messiaen dans Mode de valeurs et d'intensité (1949) et poursuivie en particulier par Boulez et par Stockhausen, a été appelée sérialisme intégral ou généralisé. Si la musique sérielle stricto sensu constitue un moment limité dans l'histoire de la musique (19201945 pour le dodécaphonisme, 1945-1955 pour le sérialisme intégral), il n'en reste pas moins que les principes sériels ont perduré jusqu'à aujourd'hui, de façon moins rigoureuse, dans ce que l'on a appelé la musique postsérielle. La musique sérielle est donc un cas particulier, systématique, de musique atonale. Par ailleurs, on peut entendre 
«atonal» dans un sens large: qui fait fi, non pas seulement du système mélodico-harmonico-métrique de la musique tonale, mais plus généralement des principes de symétrie, de pulsation rythmique régulière, d'opposition structurelle entre consonance et dissonance. Ainsi, n'est pas atonale, non seulement la musique que certains appellent néoromantique ou néo-expressionniste (en France, avec des compositeurs comme Escaich, Bacri, Tanguy, etc.), mais aussi la musique minimaliste américaine (musique répétitive de Glass, Reich, Adams, etc.)... Est atonale, au contraire, la musique sérielle et post-sérielle, mais aussi la musique concrète... La musique spectrale (abordée rapidement plus bas) pose, de ce point vue, un problème de classement.

\section{Triple entente}

Autre mise au point: certains se seront peut-être interrogés sur la pertinence de la tripartition sémantique du mot «entente» établie plus haut: "perception, compréhension et goût». Aussi ces termes - et surtout leurs relations - nécessitent-ils des explications. Tout d'abord, on peut percevoir des choses sans les comprendre (il s'agit alors d'une perception intuitive ou inconsciente); d'autre part, on peut comprendre des choses sans les percevoir (intellection pure, abstraite, théorique); enfin, on peut percevoir et/ou comprendre des choses sans qu'intervienne la question du goût. Ce troisième sens est problématique en ce qu'il introduit une dimension de jugement esthétique, mais paraît légitime. Tout d'abord, on peut considérer la notion d'esthétique, conformément à son étymologie, comme ce qui concerne la sensation, donc l'effet - sans jugement de valeur; d'autre part, il n'est pas totalement illégitime de se poser la question du plaisir esthétique. Certes, la musique, pas plus que n'importe quel autre art, n'a pas pour seule vocation de plaire; toutefois, on peut s'interroger sur l'éviction de l'affect au profit du seul intellect, dans l'avant-garde des années 1950, dans une logique purement formaliste, antiromantique par volonté de s'affranchir de l'affect, de l'expressivité, c'est-à-dire de la symbolisation.

Ces préalables (nécessaires et nécessairement schématiques) ayant été posés, venons-en à l'examen de la question: celle de la difficulté d'écoute de la musique sérielle (distinguons pour l'instant son cas de celui de la musique atonale non sérielle). La musique sérielle s'est 
développée contre les principes qui fondaient la musique tonale. Il s'agissait, en effet, pour les avant-gardistes des années 1950, d'éviter systématiquement motifs mélodiques identifiables, pulsation rythmique, répétition, hiérarchie, alternance de tensions et de détentes harmoniques. Ce parti pris empêche-t-il cette musique d'être perçue?

\section{Imperceptibilité des séries}

Lerdahl, s'appuyant notamment sur les expériences en psychologie de l'audition menées par Robert Francès ${ }^{18}$, montre, à partir de l'exemple du Marteau sans maître de Boulez, que l'impossibilité pratique de percevoir les séries (de hauteurs, de durées, d'intensités, de timbres) provient d'un hiatus entre ce qu'il appelle la «grammaire compositionnelle» et la «grammaire de l'audition». Plus précisément, cette impossibilité cognitive est due, selon lui, au fait que la musique sérielle, par opposition à la musique tonale (au sens large où l'on peut la définir), privilégie la permutation sur l'élaboration, rejette comme donnée du système l'opposition entre consonance et dissonance (laquelle serait une réalité perceptive attestée par tous les tests de psychologie cognitive) et ne permet pas de repérage cognitivement pertinent des hauteurs. Ainsi, «en ne tenant pas compte des distinctions sensorielles, le sérialisme crée des contextes musicaux qui ne sont pas appréhendés hiérarchiquement» et, "parce qu'on ne peut inférer de hiérarchies, les séries et les combinaisons sont difficiles à saisir». Lerdahl en tire alors les conclusions, ou plutôt les «revendications» esthétiques suivantes: " $1^{\circ}$ La meilleure musique utilise pleinement le potentiel de nos ressources cognitives »; « $2^{\circ}$ La meilleure musique est issue d'une alliance entre grammaire compositionnelle et grammaire auditive $»^{19}$.

\section{Critique de la critique antisérielle}

Cette analyse de Lerdahl ${ }^{20}$, l'une des plus poussées qui existent en la matière, ne laisse pas de poser un certain nombre de problèmes. Tout

18. Il montre, dans La Perception de la musique (1958, p. 218), que « l'identification des structures sérielles à la seule audition de l'œuvre était hautement improbable. » (cité par Jean-Jacques Nattiez, art. cité, p. 150).

19. Fred Lerdahl, art. cité, p. 27 et suivantes.

20. Fred Lerdahl indique être compositeur. J'avoue ne pas le connaître comme tel, et avoir fait le choix de ne pas chercher à savoir à quelle école il se rattachait, pour conserver une entière liberté de 
d'abord dans ses objectifs: que les séries ne soient pas perceptibles, même par des musiciens hautement professionnels, n'invalide en rien la musique sérielle. Jean-Jacques Nattiez fait d'ailleurs remarquer, dans le même numéro de la revue Contrechamps, que « les expériences de Francès ${ }^{21}$ démontrent la difficulté pour des personnes musicalement très éduquées de repérer les diverses apparitions du sujet et du contresujet dans deux fugues de $\mathrm{Bach}^{22}$ ». Lerdahl le reconnaît lui-même : « Je ne prétends pas non plus que les auditeurs n'infèrent aucune structure à partir des surfaces musicales composées avec des techniques sérielles. Ce que les auditeurs infèrent en fait de ces surfaces est une question intéressante qui mérite une théorie et une expérimentation propres. Mais ce n'est pas le but visé ici. » Dès lors, son objectif apparaît certes comme prudent (il s'en tient à cette absence de perception), mais aussi comme foncièrement insuffisant pour son argumentation. Problèmes également dans les présupposés et la méthode: la critique de Lerdahl à l'encontre du sérialisme repose sur l'argument de la nature. Ayant distingué grammaires compositionnelles «naturelle» et «artificielle», Lerdahl affirme, en effet, que «le problème commence seulement quand la grammaire artificielle perd contact avec la grammaire auditive $^{23} »$. Sur le plan de la méthode, Lerdahl reconnaît la part empirique (donc arbitraire) de son travail: «Pratiquement, la théorie [la sienne et celle de Jackendorff] se concentre sur la musique tonale classique, mais la plupart des règles semblent avoir une base psychologique plus générale et être ainsi tout aussi applicables à d'autres idiomes. Le degré d'universalité de ces règles est une question empirique.» Enfin, les conclusions, même rebaptisées «revendications », posent le problème du saut esthétique. Je me suis déjà prononcé sur cette référence à l'esthétique, qui n'est pas illégitime en soi : le problème, ici, est l'inadéquation entre l'objectif (la méconnaissance des séries à l'écoute de la musique sérielle) et la conclusion, ce qui me fait parler de saut. Lorsque Lerdahl « revendique» que «la meilleure musique utilise pleinement le

\footnotetext{
jugement concernant ses réflexions théoriques. On peut à bon droit supposer qu'il pratique une composition moderne (non explicitement tonale, au sens cette fois-ci de néo-tonale, harmoniquement parlant), mais fondée sur les principes tonals au sens large auxquels il fait référence en relation avec la « grammaire auditive » (sous-entendue naturelle).

21. Robert Francès, op. cit., p. 229-246.

22. Jean-Jacques Nattiez, art. cité, p. 150.

23. Art. cité, p. 30.
} 
potentiel de nos ressources cognitives » et que «la meilleure musique est issue d'une alliance entre grammaire compositionnelle et grammaire auditive », il formule une hypothèse assez arbitraire et fait reposer son argumentation sur un présupposé contestable.

\section{L'argument naturaliste}

Le vrai problème, sous-jacent à l'analyse de Lerdahl et qui doit être identifié comme un présupposé idéologique, est celui de la relation qui existe entre principes tonals, structures cognitives et nature. Si l'on identifie les deux premiers, ou en tout cas qu'on inclut (ou fait reposer) les principes tonals dans (sur) les structures cognitives de l'audition, et en les rapportant implicitement à la nature, ou du moins à des potentialités naturelles mises en œuvre culturellement, alors on verrouille la réflexion. En effet, on obtient les propositions syllogistiques suivantes (il s'agit de paraphrases, que je n'emprunte pas directement à Lerdahl) :

La musique tonale repose sur les principes naturels de la cognition humaine; or, ce n'est pas le cas de la musique sérielle; donc cette dernière n'est pas naturelle.

Mieux :

L'oreille ne peut percevoir que ce qui est rendu perceptible par les structures de la cognition; celles-ci ont des limites naturelles. La musique tonale s'est fondée sur les principes naturels de la cognition, donc elle est cognitivement adéquate, c'est-à-dire perceptible (et mémorisable). Or, la musique sérielle (ou atonale, à partir du sens large de «tonal») n'est pas fondée sur les principes naturels de la cognition, donc (et c'est là la conclusion stratégique de cette argumentation) elle n'est pas perceptible, donc (conséquence esthétique majeure) disqualifiée en tant qu'art.

Ces argumentations, que l'on pourrait appeler naturalistes, ne tiennent que par des présupposés éminemment discutables: celui de l'identification du système tonal avec les structures cognitives (à moins qu'il ne s'agisse, à l'inverse, d'une identification des structures cognitives avec le système tonal, par un effet d'illusion qui serait dû à une acculturation prolongée), et celui de la nécessité d'une audition «naturelle». Il est à cet égard caractéristique que Lerdahl, qui distingue de façon 
intéressante grammaires compositionnelles naturelle et artificielle, ne transpose pas cette distinction pour la grammaire d'écoute: or, pourquoi n'y aurait-il pas une grammaire d'écoute artificielle, comme il y en a une naturelle? On peut très bien concevoir que certains phénomènes musicaux ne se perçoivent pas à la simple écoute, même répétée, mais ne puissent se comprendre qu'à la lecture de la partition - autrement dit qu'on ne puisse pas les entendre au sens de "percevoir », mais uniquement au sens de «saisir par l'intellect» (c'est ce que reproche implicitement Robert Francès à la musique sérielle : «L'unité sérielle est d'ordre conceptuel plutôt que d'ordre perceptif ${ }^{24} \gg$ ). Pourquoi ne pas considérer que toute musique savante écrite repose sur non pas seulement une dualité, mais une dialectique entre l'écrit et le sonore? Certains diront sans doute que le résultat sonore est, dans certains cas, sans intérêt (preuve à charge : Stockhausen est allé jusqu'à revendiquer, dans les années 1950, ce désintérêt pour le résultat sonore), mais il s'agit là d'un jugement de goût qui n'est aucunement universalisable.

Touchant cette question de la nature, le rapprochement du sérialisme avec le courant, contemporain, de la musique concrète est révélateur. À la différence des compositeurs sériels, les inventeurs de la musique concrète, au premier rang desquels Pierre Schaeffer ${ }^{25}$, désireux d'explorer une nouvelle réalité sonore, font un travail électroacoustique à partir de sons naturels, concrets. La «sérialisation» de la musique concrète par Boulez (qui, comme Messiaen, travaille au Centre de musique concrète en 1952, pour y réaliser deux Études de musique concrète), a impliqué un détournement de son matériau et de sa grammaire compositionnelle: aux sons naturels de la musique concrète, le compositeur sériel substitue le matériau entièrement artificiel du signal électro-acoustique, contribuant à fonder la musique électronique. De fait, l'écoute de ces Études de Boulez provoque une difficulté accrue par rapport à ses œuvres instrumentales ou vocales (même utilisant l'électronique), en raison de l'artificialité absolue du matériau. Il reste, je le répète, que la difficulté d'une écoute naturelle, même attentive, ne dévalorise pas, en soi, cette musique.

24. Op. cit., p. 144.

25. Auteur du célèbre Traité des objets musicaux. Essai interdisciplines (Paris, Seuil, 1966), Pierre Schaeffer est, avec Pierre Henry, le fondateur du Groupe de musique concrète (1949), devenu en 1958 le Groupe de recherches musicales (GRM). 
Par ailleurs, la comparaison avec les musiques non occidentales (populaires ou savantes) ne permet ni de confirmer, ni d'infirmer le bien-fondé du présupposé naturaliste. L'examen d'un certain nombre d'entre elles (musiques noire africaine, chinoise, japonaise, balinaise, indienne, vietnamienne, etc.) tendrait, au premier abord, à conforter l'idée d'une adéquation entre grammaire compositionnelle (ici le plus souvent orale) et grammaire auditive. Nonobstant la complexité, notamment rythmique, de certaines de ces musiques (telle celle des polyphonies pygmées), qui rend difficile leur perception détaillée pour les oreilles occidentales même les plus exercées, il semble, en effet, qu'elles répondent cependant à une grammaire d'écoute naturelle, certes informée par une culture donnée. Pourtant, Sloboda, s'intéressant à la question des «universaux musicaux», vient implicitement nuancer cette assertion. Ainsi, après avoir montré, à partir des travaux ethnomusicologiques les plus sérieux, la pertinence apparemment universelle des notions de hauteur de référence (polarité dominante d'une note sur une échelle donnée) et de temps de référence (phénomène d'accentuation sur une chaîne de pulsations régulières), le psychologue cognitiviste juge ce dernier phénomène «d'[une] importance essentielle pour la musique de quasi toutes les cultures ${ }^{26}{ }_{\gg}$ : l'exception suggérée par l'adverbe (ici souligné) rend de nouveau très fragile, donc plus que problématique, l'argument d'universalité naturelle ${ }^{27}$.

\section{Musiques (d')aujourd'hui ${ }^{28}$}

Il serait pour le moins imprudent de généraliser ce qui peut être dit de la musique sérielle (d'ailleurs, on l'a vu, fort problématique) à l'en-

26. John A. Sloboda, L'Esprit musicien. La Psychologie cognitive de la musique, op. cit., p. 353 (c'est moi qui souligne).

27. Malheureusement, Sloboda ne donne pas davantage de précisions sur ce point, mais on peut supposer que cette prudence, qui ne semble pas motivée ici par une visée systématique ou par une thèse particulière de l'auteur, renvoie à (au moins) une exception effective. N'étant pas un spécialiste en ethnomusicologie, je m'en remets à lui sur ce point, me fondant sur cette nuance de Sloboda, qui m'a paru significative, sans chercher à en trouver confirmation dans les ouvrages spécialisés. Il n’y a pas là, en effet, d'enjeu capital: quand bien même (ce qui ne semble pas être le cas) aucune musique non occidentale ne sortirait du cadre d'une grammaire d'écoute naturelle (et l'on a vu combien cette notion même, n'en déplaise aux cognitivistes, était sujette à caution), le fait n'invaliderait en rien la possibilité ni, le cas échéant, la légitimité d'une grammaire d'écoute non naturelle.

28. Cet intertitre fait allusion à l'ouvrage de Pierre Boulez intitulé Penser la musique aujourd' hui (Mayence, Gonthier, 1963), dans lequel le compositeur esquisse une justification psycho-acoustique 
semble de la musique atonale. Même si l'on définit cette dernière par opposition aux principes de symétrie, de pulsation rythmique régulière et de couple structurel consonance/dissonance, elle ne constitue en aucun cas un groupe homogène. Cela étant dit, qu'en est-il, aujourd'hui, de la relation entre composition et audition? Où en sont les différents types de musique - sérielle, atonale, tonale - par rapport aux principes cognitifs de l'audition?

Tout d'abord, on remarque qu'aujourd'hui la plupart des compositeurs postsériels (et Boulez lui-même) réintroduisent, dans leurs dernières œuvres, des éléments tonals au sens large, en particulier à travers la répétition. Au sein de la musique atonale non sérielle, il en va cependant différemment, par exemple, de la musique dite «acousmatique », héritière de la musique concrète : si cette dernière constituait une exploration du domaine de l'acoustique, la musique acousmatique peut se lire comme un approfondissement de cette exploration, au moyen de celle des structures perceptives et cognitives, non sans relation avec la musique spectrale (abordée au paragraphe suivant), mais sans référence aux critères tonals ${ }^{29}$. Jean-Jacques Nattiez note la relation problématique existant entre musique acousmatique, sérialisme des années 1950 et musique électronique : « Menger $^{30}$ souligne (p. 218) que le malaise de l'auditeur face à la musique électro-acoustique a été analogue à celui créé par le sérialisme généralisé et par le post-sérialisme. Or, n'est-il pas paradoxal que la musique acousmatique se soit, en partie, développée contre le sérialisme des années cinquante, et qu'elle ait revendiqué la production d'œuvres dont les objets sonores constituants soient immédiatement perceptibles comme tels ${ }^{31}$ ?» Si le malaise évoqué par le sociologue Pierre-Michel Menger ne vient pas

\footnotetext{
de la série: «La série est, de façon très générale, le germe d'une hiérarchisation fondée sur certaines propriétés psycho-physiologiques acoustiques, douées d'une plus ou moins grande sélectivité, en vue d'organiser un ensemble FINI de possibilités créatrices liées entre elles par des affinités prédominantes par rapport à un caractère donné $[\ldots]$ » (op. cit., p. 63; c'est l'auteur qui souligne). Comme le remarque Jean-Jacques Nattiez (art. cité, p. 150), Boulez parle ici « plus que rapidement des fondements psycho-acoustiques de la série »...

29. Voir, sur la musique acousmatique, le recueil d'écrits du compositeur François Bayle, principal représentant et théoricien de ce courant: Musique acousmatique. Propositions......positions, Paris, Buchet/Chastel, Ina-GRM, 1993.

30. Référence à l'ouvrage de Pierre-Michel Menger, Le Paradoxe du musicien (Paris, Flammarion, 1983), que critique Jean-Jacques Nattiez tout au long de son article (art. cité, voir supra, note 14). 31. Art. cité, p. 150.
} 
de la proximité avec le sérialisme, ni de l'impossibilité de reconnaître immédiatement les objets sonores, viendrait-il alors de la technique électro-acoustique, qui serait perçue comme un médium ou comme un matériau trop artificiels, ou encore de l'absence de référence à la grammaire tonale - laquelle, soulignons-le, ne peut être tout uniment considérée comme naturelle? Le cas, on le voit, est complexe; encore faut-il souscrire au diagnostic du sociologue selon lequel il y aurait effectivement un «malaise»d'écoute (s'agissant des musiques électroniques, leur développement dans la musique populaire occidentale semble en tout cas rencontrer un succès exempt de ce type de «malaise»).

Par ailleurs, l'ensemble des courants tonals repose, très logiquement, sur un ou plusieurs de ces critères définitoires de la musique tonale. Ainsi, outre les musiques explicitement tonales, on remarque que la musique répétitive exacerbe principalement celui de la pulsation rythmique (l'exemple culminant en étant Music for 18 Musicians de Steve Reich). Il en va de même pour la «nouvelle simplicité» d'un Arvo Pärt, ici plutôt sur le plan des échelles modales et des intervalles. Un mot aussi du courant dit spectral (Gérard Grisey, Tristan Murail...): à l'inverse de la musique sérielle et sans doute en réaction contre elle, celui-ci a travaillé la problématique de la perception du son, d'ailleurs en référence explicite aux recherches dans le domaine de l'acoustique - certains compositeurs comme Jean-Claude Risset intégrant même à leurs œuvres des jeux d'illusions perceptives.

Bref, il semble bien qu'aujourd'hui les recherches, multiples et diverses, au sein de la musique savante ont en commun une large réintroduction de principes liés à une grammaire compositionnelle tonale.

\section{Conclusions provisoires}

\section{Doutes}

Qu'en est-il, en définitive, de ces œuvres sérielles appartenant maintenant à l'histoire de la musique - en particulier du Marteau sans maître, Structures ou des trois Sonates pour piano de Boulez, ou encore des Klavierstücke de Stockhausen? Malgré le temps (près d'un demisiècle), il apparaît que l'immense majorité des auditeurs continue d'être 
incapable d'entendre, de comprendre et de goûter ces œuvres. Sans apporter à ce problème de réponse définitive, on dira que ces dernières semblent pour l'instant excéder, pour beaucoup, nos capacités cognitives de reconnaissance et de mémorisation, ce qui ne permet en aucun cas de les invalider en tant qu'œuvres - les jugements de goût restant, quant à eux, éminemment subjectifs. Ainsi, il est très difficile de trancher la question des limites cognitives qui invalideraient la musique sérielle.

Qu'on veuille bien excuser le caractère aussi nuancé, voire mitigé, de mes conclusions quant aux problèmes perceptifs qu'entraîne la musique sérielle : à vrai dire, mes goûts m'auraient volontiers porté vers une disqualification esthétique de cette musique, ainsi que d'une partie de la musique atonale postsérielle; mais l'examen rationnel de quelques aspects de la question m'a retenu de le faire, à mon propre étonnement. Finalement, en ces temps de réaction antisérielle, qui voient un violent retour du refoulé - retour de la nécessité (naturelle) tonale, évacuée non moins violemment par la nécessité (historique) sérielle - j'aurai eu un certain plaisir, sinon à me faire l'avocat du diable, du moins à ne pas faire, en juge, la part de Dieu et l'œuvre du diable.

\section{Problématique nature}

La référence à la nature est apparue comme un nœud problématique central, non seulement pour la question abordée ici, mais plus profondément, me semble-t-il, pour les sciences cognitives dans leur ensemble. En effet, vouloir rechercher les structures de la connaissance humaine, c'est chercher à la fois quelles sont les actualisations culturelles des compétences humaines, mais aussi et surtout, par-delà la diversité de ces actualisations, quel est l'invariant de la variation, autrement dit les structures humaines naturelles, en général - l'étude des processus d'apprentissage s'inscrivant dans cette même dialectique de l'inné et de l'acquis, sans que soit opéré de façon nette le départ entre les deux pôles. De fait, les sciences cognitives associent de façon extrêmement problématique des sciences naturelles (la physiologie, la neurobiologie, etc.) et des sciences humaines (en particulier l'ethnologie et la sociologie). J'ai ici limité les références à cette question de l'inné et de l'acquis, afin de ne pas compliquer encore le problème; mais cette question demeure sous-jacente à travers la référence, 
éminemment problématique, à la nature. Notons d'ailleurs que l'ordinateur joue ici un rôle ambivalent: s'il constitue pour les sciences cognitives, sous l'aspect particulier de l'intelligence artificielle, un modèle permettant d'aider à comprendre les mécanismes cognitifs d'écoute, certaines de ses utilisations dans la composition entraînent, au contraire, une dissociation entre composition et audition - en se fondant sur des calculs abstraits a priori, qui ne reposent plus sur un contrôle, par l'oreille, du résultat sonore, la musique peut parfois dissocier grammaire compositionnelle et grammaire d'écoute, mais d'une façon qui n'est pas nécessairement négative. D'une part, il n'est pas absolument indispensable que l'auditeur entende ce que voulait lui faire entendre le compositeur (quand est-ce le cas d'ailleurs?): en ce sens, les compositeurs sériels ont peut-être créé, à leur corps (ou à leur esprit) défendant, des représentations non prévues (d'où peut-être le recours, par la suite, à l'aléatoire, qui thématise cette absence de maîtrise). D'autre part, on peut concevoir une grammaire d'écoute artificielle (et laquelle ne l'est pas, en partie?), fondée, non sur la seule audition sensible, mais sur une dialectique du sonore et de l'écrit, du phénomène et du noumène, de l'immédiat (naturel) et du médiat (culturel).

\section{BIBLIOGRAPHIE SÉLECTIVE}

Bigand Emmanuel, McAdams Stephen, éd., Penser les sons. Psychologie cognitive de l'audition, Paris, Puf, coll. «Psychologie et sciences de la pensée », 1994.

FRANCÈs Robert, La Perception de la musique, Paris, Vrin, coll. «Études de psychologie et de philosophie», 2e éd. 1984 [1958].

LERDAHL Fred, «Contraintes cognitives sur les systèmes compositionnels », dans Contrechamps, ${ }^{\circ} 10:$ "Composition et perception», 1989, p. 25-57.

DeliÈGE Irène, McAdams Stephen, éd., La Musique et les sciences cognitives, Liège et Bruxelles, Mardaga, coll. «Psychologie et sciences humaines », 1989. 


\section{Labyrinthe, $n^{\circ} 20$}

Pineau Marion, Tillmann, Barbara, Percevoir la musique: une activité cognitive, Paris, L'Harmattan, coll. «Sciences de l'éducation musicale», 2001.

Sloboda John A., L'Esprit musicien. La Psychologie cognitive de la musique, traduit en français par Marie-Isabelle Collart, Liège et Bruxelles, Mardaga, coll. «Psychologie et sciences humaines», 1988 [éd. orig. angl.: The Musical Mind, Oxford, Oxford University Press, 1985]. 